\title{
Variational Inequalities for Modeling Auction Markets with Price Map- pings
}

\author{
I.V. Konnov*
}

Department of System Analysis and Information Technologies, Kazan University, and Informatics Problems Institute, Kazan, Russia

\begin{abstract}
We propose several new models for separate and spatially distributed auction based markets where participants' reactions are described by general price mappings and show that they admit equivalent variational inequality formulations. This approach is utilized for the derivation of existence and uniqueness results and for construction of efficient solution methods.
\end{abstract}

Keywords: Auction market models, price mappings, distributed systems of auction markets, variational inequalities, solution methods.

\section{INTRODUCTION}

In comparison with the classical perfectly (Walrasian) and imperfectly (Cournot - Bertrand) competitive models, auction market models are paid considerably less attention and usually restricted with several game-theoretic models; see e.g. $[1,2]$ and references therein. However, the auction principle appears to be very suitable for control of certain economic processes related to privatization of great parts of the state property, especially, related to natural monopolies. For this reason, creation of adequate mathematical models of auction markets, which allow for investigation and solution of these very complicated problems, is very urgent.

Recently, the variational inequality approach for modeling separate auction markets of a homogeneous commodity with participants possessing price functions was proposed in [3-5]. It appeared to be very suitable both for obtaining existence and uniqueness results and for developing new solution methods.

In this paper, we first extend this approach for separate auction markets where participants' reactions are described by general multi-valued price mappings. Next, we consider an essentially more complicated problem of managing a system of such spatially separated auction markets under joint capacity and balance constraints. Such problems draw close attention due to the necessity to handle many problems arising from restructuring large energy systems. Usually, the corresponding models admit only fixed prices, nevertheless, they are formulated as two-level global optimization or socalled MPEC problems or mixed integer optimization problems which create certain difficulties in dealing with highdimensional problems arising typically in applications; see e.g. $[6,7]$ and references therein. Unlike these approaches,

*Address correspondence to this author at the Department of System Analysis and Information Technologies, Kazan University, and Informatics Problems Institute, Kazan, Russia. E-mail: konn-igor@yandex.ru we propose a variational inequality problem on a polyhedral feasible set and show that its solutions enable us to find both the bid and offer volumes and auction clearing prices for each separate market. This result gives an efficient tool for investigation and solution of very general spatial auction market problems.

\section{Single Auction with Price Mappings}

Let us consider the auction market which involves $m$ sellers and $l$ buyers of a homogeneous commodity, whose prices depend on offer/bid values. The $i$-th seller chooses his offer value $x_{i}$ within some segment $\left[\alpha^{\prime}{ }_{i}, \beta^{\prime}{ }_{i}\right]$ and his price $g_{i}$, whereas the $j$-th buyer chooses his bid value $y_{j}$ within some segment $\left[\alpha^{\prime \prime}{ }_{j}, \beta^{\prime \prime}{ }_{j}\right]$ and his price $h_{j}$. The standard situation corresponds to the case when $\alpha^{\prime}{ }_{i}=0$.

The simplest behavior of participants can be reflected by the assumption that all the prices are fixed, i.e. they are independent of volumes and choices of other participants. However, we intend to consider the more general and real case where the behavior of participants is rather complex and mutually dependent. Namely, we suppose that given an of$\mathrm{fer} / \mathrm{bid}$ values vector $(x, y)$ where $x=\left(x_{1}, \ldots, x_{m}\right)$ and $y=\left(y_{1}, \ldots, y_{l}\right)$, their price reactions constitute a set $W(x, y) \subset R^{m+l}$. This means that each price $g_{i}$ or $h_{j}$ is a function whose values depend on all the volumes and the other prices, hence

$$
w \in W(x, y) \Rightarrow w=\left(g_{1}, \ldots, g_{m}, h_{1}, \ldots, h_{l}\right) \in R^{m+l} .
$$

The particular case where prices of sellers do not depend on volumes and prices of buyers and, conversely, prices of buyers do not depend on volumes and prices of sellers, corresponds to the separable values of $W$ : 
$W(x, y)=G(x) \times H(y) ; g \in G(x), h \in H(y)$

$\Rightarrow g=\left(g_{1}, \ldots, g_{m}\right), h=\left(h_{1}, \ldots, h_{l}\right)$.

In the single-valued case we simply have

$W(x, y)=\left\{\left(g_{1}(x, y), \ldots, g_{m}(x, y), h_{1}(x, y), \ldots, h_{l}(x, y)\right)\right\}$.

In general, we thus define the price mapping $W: R^{m+l} \rightarrow \Pi\left(R^{m+l}\right)$. Here and below, $\Pi(A)$ denotes the family of all nonempty subsets of a set $A$. Additionally, we can take into account the "passive" economic agents who do not participate explicitly in the auction process but agree beforehand with its price. We suppose that their total excess demand is fixed and equal to $b$. The value of $b$ may be positive or negative and may in principle determine the prescribed dis-balance value. The usual choice $b=0$ leads to the precise balance and forces the auction market to be a closed system. However, if $b$ is an arbitrary parameter, we can place the model in more general settings and take into account the reaction of some external economic agents. The solution of the problem is constituted by a volumes vector $\left(x^{*}, y^{*}\right)$ and a price $p^{*}$ such that there exists

$w^{*}=\left(g_{1}^{*}, \ldots, g_{m}^{*}, h_{1}^{*}, \ldots, h_{l}^{*}\right) \in W\left(x^{*}, y^{*}\right)$,

satisfying the conditions:

$$
g_{i}^{*}\left\{\begin{array}{l}
\geq p^{*} \quad \text { if } x_{i}^{*}=\alpha^{\prime}{ }_{i}, \\
=p^{*} \quad \text { if } x_{i}^{*} \in\left(\alpha^{\prime}{ }_{i}, \beta^{\prime}{ }_{i}\right), \quad \text { for } \quad i=1, \ldots, m ; \\
\leq p^{*} \quad \text { if } x_{i}^{*}=\beta^{\prime}{ }_{i},
\end{array}\right.
$$

and

$$
h_{j}^{*}\left\{\begin{array}{lc}
\leq p^{*} & \text { if }_{j}^{*}=\alpha^{\prime \prime}, \\
=p^{*} & \text { if } y_{j}^{*} \in\left(\alpha^{\prime \prime}, \beta_{j}^{\prime \prime}\right), \quad \text { for } \quad j=1, \ldots, l ; \\
\geq p^{*} & {\text { ify }{ }_{j}^{*}=\beta^{\prime \prime}}_{j},
\end{array}\right.
$$

and also

$$
\left(x^{*}, y^{*}\right) \in Z \text {, }
$$

where

$$
Z=\left\{\begin{array}{c|c}
\sum_{i=1}^{m} x_{i}-\sum_{j=1}^{l} y_{j}=b, \\
\alpha^{\prime}{ }_{i} \leq x_{i} \leq \beta^{\prime}, i=1, \ldots, m, \\
\alpha^{\prime \prime}{ }_{j} \leq y_{j} \leq \beta^{\prime \prime}{ }_{j}, j=1, \ldots, l .
\end{array}\right\} .
$$

Thus, the choice of bid/offer volumes must be feasible in the sense of restrictions for volumes of economic agents and equilibrate the supply and demand, furthermore, each trader sells the minimal (respectively, maximal) value if its price is greater (less) than the (unknown) auction price $p^{*}$, and each buyer purchases the maximal (respectively, minimal) value if its price is greater (less) than the (unknown) auction price $p^{*}$, which conforms to the auction principle.
The main difficulty of the formulation (1)-(3) is in the fact that it involves the superfluous unknown auction price. We propose an equivalent variational inequality (VI) formulation of the problem for excluding the unknown price $p^{*}$.

Theorem 1. (i) If $\left(x^{*}, y^{*}, p^{*}\right)$ is a solution of problem (1)(3), then $\left(x^{*}, y^{*}\right)$ solves the problem:

$$
\begin{gathered}
\exists w^{*}=\left(g_{1}^{*}, \ldots, g_{m}^{*}, h_{1}^{*}, \ldots, h_{l}^{*}\right) \in W\left(x^{*}, y^{*}\right), \\
\sum_{i=1}^{m} g_{i}^{*}\left(x_{i}-x_{i}^{*}\right)-\sum_{j=1}^{l} h_{j}^{*}\left(y_{j}-y_{j}^{*}\right) \geq 0 \quad \forall(x, y) \in Z .
\end{gathered}
$$

(ii) Conversely, if $\left(x^{*}, y^{*}\right) \in Z$ satisfies (4), then there exists a number $p^{*}$ such that $\left(x^{*}, y^{*}, p^{*}\right)$ is a solution of problem (1)-(3).

Proof. (i) Let (1)-(2) hold for some $\left(x^{*}, y^{*}\right) \in Z$ and $w^{*}=\left(g_{1}^{*}, \ldots, g_{m}^{*}, h_{1}^{*}, \ldots, h_{l}^{*}\right) \in W\left(x^{*}, y^{*}\right)$. Then we can define the Lagrangian

$$
L(x, y, p)=\sum_{i=1}^{m} g_{i}^{*} x_{i}-\sum_{j=1}^{l} h_{j}^{*} y_{j}-p\left(\sum_{i=1}^{m} x_{i}-\sum_{j=1}^{l} y_{j}-b\right)
$$

and rewrite conditions (1)-(2) as follows:

$$
\begin{array}{cc}
\frac{\partial L\left(x^{*}, y^{*}, p^{*}\right)}{\partial x_{i}}\left(x_{i}-x_{i}^{*}\right) \geq 0 & \forall x_{i} \in\left[\alpha^{\prime}{ }_{i}, \beta_{i}^{\prime}\right], \quad i=1, \ldots, m ; \\
\frac{\partial L\left(x^{*}, y^{*}, p^{*}\right)}{\partial y_{j}}\left(y_{j}-y_{j}^{*}\right) \geq 0 & \forall y_{j} \in\left[\alpha^{\prime \prime}{ }_{j}, \beta^{\prime \prime}{ }_{j}\right], \quad j=1, \ldots, l .
\end{array}
$$

By using the suitable Karush-Kuhn-Tucker theorem (see e.g. [8, Chapter 4, Theorem 2.4] or [9, Proposition 1.3.4]), we see that $\left(x^{*}, y^{*}\right)$ must solve the problem

$\operatorname{minimize} \quad \sum_{i=1}^{m} g_{i}^{*} x_{i}-\sum_{j=1}^{l} h_{j}^{*} y_{j}$,

$(x, y) \in Z$

i.e. $\left(x^{*}, y^{*}\right)$ solves problem (4).

(ii) If a pair $\left(x^{*}, y^{*}\right)$ solves problem (4) for some $w^{*}=\left(g_{1}^{*}, \ldots, g_{m}^{*}, h_{1}^{*}, \ldots, h_{l}^{*}\right) \in W\left(x^{*}, y^{*}\right)$, it solves (7). By using the other part of the same Karush-Kuhn-Tucker theorem, we obtain that there exists $p^{*}$ such that (6) holds, i.e. the Lagrangian defined in (5) has the saddle point. But (6) implies (1)-(2) and the result follows.

From the proof it follows that the auction price $p^{*}$ coincides with the Lagrange multiplier for the balance constraint $\sum_{i=1}^{m} x_{i}-\sum_{j=1}^{l} y_{j}=b$. After solving VI (4) we can find the auction price easily from (1)-(2).

Observe that each participant, unlike the perfect competition conditions, may now utilize additional information about the other agents, however, the auctioneer rule gives a 
clear principle for setting the price. If we define the mapping $V: R^{m+l} \rightarrow \Pi\left(R^{m+l}\right)$ by the formula:

$$
\begin{gathered}
v \in V(x, y) \Longleftrightarrow v=\left(v_{1}, \ldots, v_{m}, v_{m+1}, \ldots, v_{m+l}\right), \\
\left(v_{1}, \ldots, v_{m},-v_{m+1}, \ldots,-v_{m+l}\right) \in W(x, y)
\end{gathered}
$$

and set $z=(x, y)$, then (4) is rewritten in the standard multivalued VI format: Find $z^{*} \in Z$ such that

$\exists v^{*} \in V\left(z^{*}\right),<v^{*}, z-z^{*}>\geq 0 \quad \forall z \in Z$.

Hence, we can apply the well-developed techniques from the theory and solution methods of VIs for investigation and solution of the initial problem.

Recall that a multivalued mapping $Q: R^{n} \rightarrow \Pi\left(R^{n}\right)$ is said to be a $K$ (Kakutani)-mapping on a set $X$ if it is upper semicontinuous on $X$ and has nonempty, convex, and compact values.

For instance, we apply the known result that any VI with $K$-mapping and convex and compact feasible set is solvable; see e.g. [10] and [11, Theorem 4.2.12].

Corollary 1. If the set $Z$ is nonempty and bounded, and $V: R^{m+l} \rightarrow \Pi\left(R^{m+l}\right)$ in (8) is a $K$-mapping on $Z$, then problem (4) is solvable.

Obviously, solvability of VI (4) (or (9)) implies the solvability of the auction equilibrium problem (1)-(3) with the corresponding feasible set. The uniqueness may be derived under the strict monotonicity of the mapping $V$. Besides, there are many other existence and uniqueness theorems for VIs, including the unbounded case (see e.g. [11,12] and references therein), which can be also applied to the above problem.

Being based on these results, we can utilize numerous iterative algorithms for VIs (see $[13,12,9]$ ) both for computation of a solution of auction market problems and for modeling dynamic auction market processes and investigating their stability.

\section{Constrained Spatial Auction Markets with Price Map- pings}

We now consider a system of $n$ markets of a homogeneous commodity, which are joined by links (transmission lines) in a network. We denote by $I_{k}$ and $J_{k}$ respectively, the index sets of sellers and buyers of the $k$-th local market associated with the $k$-th node. It is supposed that the $i$-th seller chooses his offer value $x_{i}$ within the segment $\left[\alpha^{\prime}{ }_{i}, \beta_{i}^{\prime}\right]$ with $\alpha^{\prime}{ }_{i} \geq 0$ for $i \in I_{k}$ and his price $g_{i}$, whereas the $j$-th buyer chooses his bid value $y_{j}$ within the segment $\left[\alpha^{\prime \prime}{ }_{j}, \beta^{\prime \prime}{ }_{j}\right]$ with $\alpha^{\prime \prime}{ }_{j} \geq 0$ and his price $h_{j}$ for $j \in J_{k}$. We also suppose that the behavior of participants of the $k$-th local market is the same as in the previous case. That is, given the volume vectors $x_{(k)}=\left(x_{i}\right)_{i \in I_{k}}$ and $y_{(k)}=\left(y_{j}\right)_{j \in J_{k}}$, their price reaction is a set $W_{(k)}\left(x_{(k)}, y_{(k)}\right)$, hence

$w_{(k)} \in W_{(k)}\left(x_{(k)}, y_{(k)}\right) \Rightarrow w_{(k)}=\left(\left(g_{i}\right)_{i \in I_{k}},\left(h_{j}\right)_{j \in J_{k}}\right)$,

i.e., it again involves prices of sellers and buyers of this market, thus defining the multi-valued mapping $\left(x_{(k)}, y_{(k)}\right) \mapsto W_{(k)}\left(x_{(k)}, y_{(k)}\right)$. We define the sets of offer/bid bounds for the $k$-th auction

$X_{(k)}=\prod_{i \in I_{k}}\left[\alpha^{\prime}{ }_{i}, \beta^{\prime}{ }_{i}\right], \quad Y_{(k)}=\prod_{j \in J_{k}}\left[\alpha^{\prime \prime}{ }_{j}, \beta^{\prime \prime}{ }_{j}\right]$.

Due to the auction principle, the solutions $\left(x_{(k)}^{*}, y_{(k)}^{*}\right) \in X_{(k)} \times Y_{(k)}$ must satisfy the auction market conditions:

$\exists w_{(k)}^{*}=\left(\left(g_{i}^{*}\right)_{i \in I_{k}},\left(h_{j}^{*}\right)_{j \in J_{k}}\right) \in W_{(k)}\left(x_{(k)}^{*}, y_{(k)}^{*}\right)$

such that

$g_{i}^{*}\left\{\begin{array}{c}\geq p_{k}^{*} \text { if } x_{i}^{*}=\alpha^{\prime}{ }_{i}, \\ p_{k}^{*} \text { if } \quad x_{i}^{*} \in\left(\alpha^{\prime}, \beta_{i}^{\prime}\right), i \in I_{k} ; \\ \leq p_{k}^{*} \text { if } x_{i}^{*}=\beta^{\prime}{ }_{i},\end{array}\right.$

and

$$
h_{j}^{*}\left\{\begin{array}{c}
\leq p_{k}^{*} \text { if } y_{j}^{*}=\alpha^{\prime \prime}, \\
=p_{k}^{*} \quad \text { if } y_{j}^{*} \in\left(\alpha^{\prime \prime}, \beta^{\prime \prime}{ }_{j}\right), j \in J_{k} ; \\
\geq p_{k}^{*} \text { if } y_{j}^{*}=\beta^{\prime \prime},
\end{array}\right.
$$

where $p_{k}^{*}$ is the (unknown) auction clearing price of the $k$ th market. Also, the solutions must satisfy the market balance equation:

$\sum_{i \in I_{k}} x_{i}^{*}-\sum_{j \in J_{k}} y_{j}^{*}-u_{k}^{*}=0$

where $u_{k}^{*}$ is the (unknown) value of external (with respect to the $k$-th market) demand and these values give the total balance equation for the system:

$\sum_{k=1}^{n} u_{k}^{*}=0$.

However, we have also to take into account the conditions of the graph associated with the system of distributed markets. We denote by $A$ the set of all the arcs joining the nodes attributed to markets. Let $f_{a}$ denote the commodity flow for arc $a=(k, l)$ and let $\left[b^{\prime}{ }_{a}, b^{\prime \prime}{ }_{a}\right]$ be the segment of feasible upper capacity bounds for this arc. Given the flow vector $f=\left(f_{a}\right)_{a \in A}$, we can define the cost $c_{a}=c_{a}(f)$ of shipment on one unit of the commodity along arc $a \in A$. 
Next, for a given node $k$, we denote by $A_{k}^{+}$and $A_{k}^{-}$the sets of incoming and outgoing arcs at $k$. Note that $I_{k}$ and $J_{k}$ can be empty for some $k$ and this case corresponds to an intermediate node.

If $f^{*}$ is the optimal flow distribution corresponding to $x_{(k)}^{*}, y_{(k)}^{*}, u_{k}^{*}$ in (11)-(14), then we have the node balance equation

$$
\sum_{a \in A_{k}^{-}} f_{a}^{*}-\sum_{a \in A_{k}^{+}} f_{a}^{*}-u_{k}^{*}=0, \quad k=1, \ldots, n
$$

and the flow capacity constraints

$$
f_{a}^{*} \in\left[b^{\prime}{ }_{a}, b^{\prime \prime}{ }_{a}\right], \quad a \in A \text {. }
$$

In principle, relations (10)-(13) for $k=1, \ldots, n$, and (14)(16) give a possible formulation of the constrained spatial equilibrium problem for a system of auction markets. However, we can impose the additional equilibrium conditions on prices, unit transmission costs, and transmission volumes between markets:

$$
c_{a}\left(f^{*}\right)+p_{k}^{*}-p_{l}^{*}\left\{\begin{array}{c}
\geq 0 \text { if } f_{a}^{*}=b^{\prime}{ }_{a}, \\
0 \text { if } f_{a}^{*} \in\left(b^{\prime}{ }_{a}, b^{\prime \prime}{ }_{a}\right), \quad \forall a=(k, l) \in A . \\
\leq 0 \text { if } f_{a}^{*}=b^{\prime \prime}{ }_{a},
\end{array}\right.
$$

Then, the basic constrained spatial auction market equilibrium problem will consist in finding $\left(x^{*}, y^{*}, u^{*}, f^{*}\right)$ satisfying (10)-(13) for $k=1, \ldots, n$, and (14)-(17), where $x^{*}=\left(x_{(k)}^{*}\right)_{k=1, \ldots, n}, y^{*}=\left(y_{(k)}^{*}\right)_{k=1, \ldots, n}, u^{*}=\left(u_{1}^{*}, \ldots, u_{n}^{*}\right)$.

We now present a variational inequality problem, whose solutions satisfy the above conditions. Set

$$
X=\prod_{k=1}^{n} X_{(k)}, Y=\prod_{k=1}^{n} X_{(k)}, F=\prod_{a \in A}\left[b_{a}^{\prime}, b^{\prime \prime}{ }_{a}\right]
$$

and define the set

$$
D=\left\{\begin{array}{c|c}
(x, y, f) & \left(\sum_{a \in A_{k}^{-}} f_{a}-\sum_{a \in A_{k}^{+}} f_{a}\right) \\
\in X \times Y \times F & -\left(\sum_{i \in I_{k}} x_{i}-\sum_{j \in J_{k}} y_{j}\right)=0 \quad k=1, \ldots, n
\end{array}\right\} .
$$

The problem is to find $\left(x^{*}, y^{*}, f^{*}\right) \in D$ such that

$$
\begin{aligned}
\exists w_{(k)}^{*}= & \left(\left(g_{i}^{*}\right)_{i \in I_{k}},\left(h_{j}^{*}\right)_{j \in J_{k}}\right) \in W_{(k)}\left(x_{(k)}^{*}, y_{(k)}^{*}\right), \quad k=1, \ldots, n ; \\
& \sum_{k=1}^{n}\left[\sum_{i \in I_{k}} g_{i}^{*}\left(x_{i}-x_{i}^{*}\right)-\sum_{j \in J_{k}} h_{j}^{*}\left(y_{j}-y_{j}^{*}\right)\right] \\
+ & \sum_{a \in A} c_{a}\left(f^{*}\right)\left(f_{a}-f_{a}^{*}\right) \geq 0 \quad \forall(x, y, f) \in D .
\end{aligned}
$$

Observe that the above VI involves only volume variables and its feasible set is clearly convex and closed. Also, instead of the separate balance equations (13) and (15), we utilize in (18) the material balance equation between external and internal flows at each market.
First we give the necessary and sufficient conditions of optimality for problem (18)-(19).

Proposition 1. (i) If $\left(x^{*}, y^{*}, f^{*}\right)$ is a solution of problem (18)-(19), there exist numbers $p_{k}^{*}, k=1, \ldots, n$ such that

$$
\begin{gathered}
\exists w_{(k)}^{*}=\left(\left(g_{i}^{*}\right)_{i \in I_{k}},\left(h_{j}^{*}\right)_{j \in J_{k}}\right) \in W_{(k)}\left(x_{(k)}^{*}, y_{(k)}^{*}\right), \quad k=1, \ldots, n ; \\
\sum_{k=1}^{n}\left[\sum_{i \in I_{k}} g_{i}^{*}\left(x_{i}-x_{i}^{*}\right)-\sum_{j \in J_{k}} h_{j}^{*}\left(y_{j}-y_{j}^{*}\right)\right] \\
+\sum_{a \in A} c_{a}\left(f^{*}\right)\left(f_{a}-f_{a}^{*}\right)-\sum_{k=1}^{n} p_{k}^{*}\left[\sum_{i \in I_{k}}\left(x_{i}-x_{i}^{*}\right)-\sum_{j \in J_{k}}\left(y_{j}-y_{j}^{*}\right)\right. \\
\left.-\sum_{a \in A_{k}^{-}}\left(f_{a}-f_{a}^{*}\right)+\sum_{a \in A_{k}^{+}}\left(f_{a}-f_{a}^{*}\right)\right] \geq 0 \quad \forall(x, y, f) \in X \times Y \times F
\end{gathered}
$$

and

$\left(\sum_{a \in A_{k}^{-}} f_{a}^{*}-\sum_{a \in A_{k}^{+}} f_{a}^{*}\right)-\left(\sum_{i \in I_{k}} x_{i}^{*}-\sum_{j \in J_{k}} y_{j}^{*}\right)=0 \quad k=1, \ldots, n$.

(ii) If elements $\left(x^{*}, y^{*}, f^{*}, p^{*}\right) \in X \times Y \times F \times R^{n}$ satisfy (20)-(21), then $\left(x^{*}, y^{*}, f^{*}\right)$ solves problem (18)-(19).

Proof. If the triplet $\left(x^{*}, y^{*}, f^{*}\right)$ is a solution of problem (18)-(19), it solves the optimization problem:

$$
\text { minimize } \quad \sum_{k=1}^{n}\left[\sum_{i \in I_{k}} g_{i}^{*} x_{i}-\sum_{j \in J_{k}} h_{j}^{*} y_{j}\right]+\sum_{a \in A} c_{a}\left(f^{*}\right) f_{a} \text {. }
$$

$(x, y, f) \in D$

Then, using the "necessity" part of the suitable KarushKuhn-Tucker theorem (see e.g. [8, Chapter 4, Theorem 2.4] or [9, Proposition 1.3.4]), we see that there exist numbers $p_{k}^{*}, k=1, \ldots, n$ such that (20)-(21) hold true.

Conversely, if elements $\left(x^{*}, y^{*}, f^{*}, p^{*}\right) \in X \times Y \times F \times R^{n}$ satisfy (20)-(21, then, by using the "sufficiency" part of the same Karush-Kuhn-Tucker theorem, we see that the triplet $\left(x^{*}, y^{*}, f^{*}\right)$ must solve the optimization problem (22), therefore, it solves problem (18)-(19).

Now we can establish the basic equivalence result.

Theorem 2. (i) If $\left(x^{*}, y^{*}, f^{*}\right)$ is a solution to (18)-(19), then there exist numbers $p_{k}^{*}$ and $u_{k}^{*}, k=1, \ldots, n$ such that (10)-(13) for $k=1, \ldots, n$, and (14)-(17) hold true.

(ii) If elements $\left(x^{*}, y^{*}, f^{*}, p^{*}, u^{*}\right) \in X \times Y \times F \times R^{n} \times R^{n}$ satisfy (10)-(13) for $k=1, \ldots, n$, and (14)-(17), then $\left(x^{*}, y^{*}, f^{*}\right)$ solves problem (18)-(19).

Proof. Due to Proposition 1 we see that it is sufficient to show that for given elements $\left(x^{*}, y^{*}, f^{*}, p^{*}\right) \in X \times Y \times F \times R^{n}$ the conditions (10)-(13) for $k=1, \ldots, n$, and (14)-(17) are equivalent to (20)-(21). So, let first the conditions (20)-(21) hold true for some elements $\left(x^{*}, y^{*}, f^{*}, p^{*}\right) \in X \times Y \times F \times R^{n}$. 
Then (10) clearly holds. Determine the numbers $u_{k}^{*}$, $k=1, \ldots, n$ from (15), then (21) gives (13). Moreover, summing (15) over $k=1, \ldots, n$ gives

$$
\sum_{k=1}^{n} u_{k}^{*}=\sum_{k=1}^{n}\left(\sum_{a \in A_{k}^{-}} f_{a}^{*}-\sum_{a \in A_{k}^{+}} f_{a}^{*}\right)=0
$$

since the right-hand side expression involves twice the flow value for each arc $a$ with opposite signs. Hence, (14) also holds. Next, we see that (20) is equivalent to the following set of partial variational inequalities:

$$
\begin{gathered}
\left(g_{i}^{*}-p_{k}^{*}\right)\left(x_{i}-x_{i}^{*}\right) \geq 0 \quad \forall x_{i} \in\left[\alpha^{\prime}{ }_{i}, \beta_{i}{ }_{i}\right], i \in I_{k}, k=1, \ldots, n ; \\
\left(p_{k}^{*}-h_{j}^{*}\right)\left(y_{j}-y_{j}^{*}\right) \geq 0 \quad \forall y_{j} \in\left[\alpha^{\prime \prime}{ }_{j}, \beta^{\prime \prime}{ }_{j}\right], j \in J_{k}, k=1, \ldots, n ; \\
\left(c_{a}\left(f^{*}\right)+p_{k}^{*}-p_{l}^{*}\right)\left(f_{a}-f_{a}^{*}\right) \geq 0 \quad \forall f_{a} \in\left[b^{\prime}{ }_{a}, b^{\prime \prime}{ }_{a}\right], \forall a=(k, l) \in A .
\end{gathered}
$$

However, these relations are equivalent to (11), (12), and (17), respectively. Therefore, assertion (i) is true.

Conversely, let elements $\left(x^{*}, y^{*}, f^{*}, p^{*}, u^{*}\right) \in X \times Y \times F \times R^{n} \times R^{n}$ satisfy (10)-(13) for $k=1, \ldots, n$, and (14)-(17). Then we have $\left(x^{*}, y^{*}, f^{*}\right) \in X \times Y \times F$ and (13), (15) yield (21). Besides, it was mentioned that (11), (12), and (17) are equivalent to (23), which, in turn, is equivalent to (20). Therefore, assertion (ii) is also true. The proof is complete.

Thus, one can find easily a solution of the spatial auction problem (10)-(17) from the solution of VI (18)-(19). The sense of problem (18)-(19) is also clear: Find the feasible triplet $\left(x^{*}, y^{*}, f^{*}\right) \in D$ such that it minimizes the total diseconomies in the system for the corresponding offer/bid prices $g^{*}$ and $h^{*}$ and for the corresponding shipment costs $c=c\left(f^{*}\right)$. Observe that we do not impose any conditions on the mappings $W_{(k)}$, i.e. on functions $g, h$, and $c$, but it would be reasonable to suppose that they have non-negative values and that the function $c$ is continuous. For instance if $c \equiv 0$, then problem (18)-(19) reflects the maximization of pure auction markets profit.

Observe that for any solution of problem (10)-(17) obtained from VI (18)-(19) the auction clearing prices $p_{k}^{*}, k=1, \ldots, n$ are Lagrange multipliers for the node balance constraints from (20).

Being based on Theorem 2, we can derive existence and uniqueness results for the spatial auction market equilibrium problem problem from the theory of variational inequalities. To this end, let us define the mapping $\left(x_{(k)}, y_{(k)}\right) \mapsto$ $V_{(k)}\left(x_{(k)}, y_{(k)}\right)$ by the formula:

$$
\begin{gathered}
v^{(k)} \in V_{(k)}\left(x_{(k)}, y_{(k)}\right) \Longleftrightarrow v^{(k)}=\left(\left(v_{i}^{\prime}\right)_{i \in I_{k}},\left(v^{\prime \prime}\right)_{j \in J_{k}}\right), \\
\left.\left(\left(v_{i}^{\prime}\right)_{i \in I_{k}},\left(-v^{\prime \prime}\right)_{j}\right)_{j \in J_{k}}\right) \in W_{(k)}\left(x_{(k)}, y_{(k)}\right),
\end{gathered}
$$

and set

$$
V(x, y)=\prod_{k=1}^{n} V_{(k)}\left(x_{(k)}, y_{(k)}\right)
$$

(cf. (8)). Then problem (18)-(19) becomes the standard VI with the underlying mapping $(x, y, f) \mapsto(V(x, y), c(f))$ and feasible set $D$ (cf. (9)).

Theorem 3. Suppose that the set $D$ is nonempty and bounded, $(x, y) \mapsto V(x, y)$ is a $K$-mapping on $D$, and that the mapping $f \mapsto c(f)$ is continuous on $D$. Then problem (18)-(19) has a solution.

In fact, (18)-(19) is then a VI with $K$-mapping and nonempty, convex, and compact feasible set and must have a solution; see e.g. [10].

Recall that a mapping $Q$ is said to be

monotone on $X$ if for each for each pair of points $x, y \in X$ and for all $q^{\prime} \in Q(x), q^{\prime \prime} \in Q(y)$, we have

$<q^{\prime}-q^{\prime \prime}, x-y>\geq 0$

(ii) strictly monotone on $X$ if for all distinct $x, y \in X$ and for all $q^{\prime} \in Q(x), q^{\prime \prime} \in Q(y)$, we have

$<q^{\prime}-q^{\prime \prime}, x-y>>0$.

Clearly, strict monotonicity of $V$ and $c$ yields the uniqueness for VI (18)-(19); see e.g. [10, Theorem 4.2]. Combining this property with Theorem 3 , we obtain also the uniqueness and existence result.

Theorem 4. Suppose that the set $D$ is nonempty and bounded, $\quad(x, y) \mapsto V(x, y)$ is a strictly monotone $K$ mapping on $D$, and that the mapping $f \mapsto c(f)$ is continuous and strictly monotone on $D$. Then problem (18)-(19) has a unique solution.

\section{Solution Methods}

Being based on the above results, we can propose various iterative solution methods for problem (18)-(19), hence, for the spatial auction market problem (10)-(16); see [13,12,9]. Of course, we are interested in methods taking into account the essential features of the problem, such as separability of constraints. For instance, under the monotonicity assumptions on $V$ and $c$ we can apply the well-known proximal point method (see [14]), which consists in generating the iteration sequence $\left\{\left(x^{s}, y^{s}, f^{s}\right)\right\}$ in conformity with the formula: Find $\left(x^{s+1}, y^{s+1}, f^{s+1}\right) \in D$ such that

$$
\begin{gathered}
\exists w_{(k)}^{s+1}=\left(\left(g_{i}^{s+1}\right)_{i \in I_{k}},\left(-h_{j}^{s+1}\right)_{j \in J_{k}}\right) \in W_{(k)}\left(x_{(k)}^{s+1}, y_{(k)}^{s+1}\right), \quad k=1, \ldots, n ; \\
\sum_{k=1}^{n}\left[\sum_{i \in I_{k}}\left(g_{i}^{s+1}+\theta_{s}^{-1}\left(x_{i}^{s+1}-x_{i}^{s}\right)\right)\left(x_{i}-x_{i}^{s+1}\right)\right. \\
\left.-\sum_{j \in J_{k}}\left(h_{j}^{s+1}-\theta_{s}^{-1}\left(y_{j}^{s+1}-y_{j}^{s}\right)\right)\left(y_{j}-y_{j}^{s+1}\right)\right] \\
+\sum_{a \in A}\left(c_{a}\left(f^{s+1}\right)+\theta_{s}^{-1}\left(f_{a}^{s+1}-f_{a}^{s}\right)\right)\left(f_{a}-f_{a}^{s+1}\right) \geq 0 \\
\forall(x, y, f) \in D ;
\end{gathered}
$$

where $\theta_{s}>0$ is the stepsize parameter. The preference of this method is that (24) has always a unique solution if the set $D$ is nonempty, i.e. under very mild assumptions. 
To illustrate further preferences of the above method, let us now consider the simple decomposable case where there exist convex differentiable functions $\mu_{i}:\left[\alpha_{i}, \beta_{i}\right] \rightarrow R$, $i \in I_{k}, \quad-\eta_{j}:\left[\alpha_{j}, \beta_{j}\right] \rightarrow R, \quad j \in J_{k}, \quad k=1, \ldots, n, \quad$ and $\sigma_{a}:\left[b_{a}^{\prime}, b^{\prime \prime}{ }_{a}\right] \rightarrow R, a \in A$, such that

$$
\mu_{i}^{\prime}\left(x_{i}\right)=g_{i}\left(x_{i}\right), \eta_{j}^{\prime}\left(y_{j}\right)=h_{j}\left(y_{j}\right), \sigma_{a}^{\prime}\left(f_{a}\right)=c_{a}\left(f_{a}\right) .
$$

This means that the price function of each seller/buyer can depend only on his offer/bid value and that, the transmission cost for each arc can depend only on its flow value. Then VI (18)-(19) becomes equivalent to the convex optimization problem:

$$
\begin{gathered}
\operatorname{minimize} \sum_{k=1}^{n}\left[\sum_{i \in I_{k}} \mu_{i}\left(x_{i}\right)-\sum_{j \in J_{k}} \eta_{j}\left(y_{j}\right)\right]+\sum_{a \in A} \sigma_{a}\left(f_{a}\right) . \\
(x, y, f) \in D
\end{gathered}
$$

In turn, VI (24) is replaced by the auxiliary optimization problem:

$$
\begin{array}{ll}
\operatorname{minimize} & \left\{\sum _ { k = 1 } ^ { n } \left[\sum_{i \in I_{k}}\left(\mu_{i}\left(x_{i}\right)+0.5 \theta_{s}^{-1}\left(x_{i}-x_{i}^{s}\right)^{2}\right)\right.\right. \\
(x, y, f) \in D & \left.-\sum_{j \in J_{k}}\left(\eta_{j}\left(y_{j}\right)-0.5 \theta_{s}^{-1}\left(y_{j}-y_{j}^{s}\right)^{2}\right)\right] \\
& \left.+\sum_{a \in A}\left(\sigma_{a}\left(f_{a}\right)+0.5 \theta_{s}^{-1}\left(f_{a}-f_{a}^{s}\right)^{2}\right)\right\}
\end{array}
$$

Obviously, the combination of the proximal point and dual ascent methods seems now rather suitable. In fact, at the $s$-th iteration, we can solve (25) via the dual problem

$\operatorname{maximize} \varphi_{\mathrm{s}}(\mathrm{p})$,

$$
p \in R^{n}
$$

where

$$
\begin{array}{r}
\varphi_{s}(p)=\min _{(x, y, f) \in X \times Y \times F}\left\{\sum _ { k = 1 } ^ { n } \left[\sum_{i \in I_{k}}\left(\mu_{i}\left(x_{i}\right)+0.5 \theta_{s}^{-1}\left(x_{i}-x_{i}^{s}\right)^{2}\right)\right.\right. \\
\left.-\sum_{j \in J_{k}}\left(\eta_{j}\left(y_{j}\right)-0.5 \theta_{s}^{-1}\left(y_{j}-y_{j}^{s}\right)^{2}\right)\right] \\
+\sum_{a \in A}\left(\sigma_{a}\left(f_{a}\right)+0.5 \theta_{s}^{-1}\left(f_{a}-f_{a}^{s}\right)^{2}\right) \\
\left.+\sum_{k=1}^{n} p_{k}\left[\left(\sum_{a \in A_{k}^{-}} f_{a}-\sum_{a \in A_{k}^{+}} f_{a}\right)-\left(\sum_{i \in I_{k}} x_{i}-\sum_{j \in J_{k}} y_{j}\right)\right]\right] \\
=\sum_{k=1}^{n}\left[\sum_{i \in I_{k}} \min _{x_{i} \in\left[\alpha_{i}, \beta_{i}\right]}\left(\mu_{i}\left(x_{i}\right)+0.5 \theta_{s}^{-1}\left(x_{i}-x_{i}^{s}\right)^{2}-p_{k} x_{i}\right)\right. \\
\left.-\sum_{j \in J_{k}} \max _{y_{j} \in\left[\alpha_{j}, \beta_{j}\right]}\left(\eta_{j}\left(y_{j}\right)-0.5 \theta_{s}^{-1}\left(y_{j}-y_{j}^{s}\right)^{2}-p_{k} y_{j}\right)\right] \\
+\sum_{a=(k, l) \in A} \min _{f_{a} \in\left[b^{\prime} a b^{\prime} a\right.}\left(\sigma_{a}\left(f_{a}\right)+0.5 \theta_{s}^{-1}\left(f_{a}-f_{a}^{s}\right)^{2}+\left(p_{k}-p_{l}\right) f_{a}\right) .
\end{array}
$$

Clearly, the computation of the values of $\varphi$ and its gradient can be made componentwise, i.e. (27) is decomposed into a set of separable one-dimensional problems. In order to solve (26) we can apply a suitable conjugate gradient method, which seems more efficient here in comparison with the usual gradient one, as in the classical Uzawa method. It should be noted that a combined proximal point and dual quasi-Newton method for separable optimization problems was considered in [15]. Similar dual techniques for more general decomposable problems were developed in [16,17]. Also, together with the classical proximal point method one can utilize somewhat more general schemes; see e.g. [18-20].

The above combined proximal point and dual conjugate gradient method for subproblems was implemented for solving several spatial auction problems. To create the model examples we took the data from real electricity market systems. In these problems, all the prices $g_{i}$ and $h_{j}$ were fixed and $c_{a} \equiv 0$. In the method, each auxiliary optimization problem (25) was solved by the dual conjugate gradient method within the fixed prescribed accuracy for the norm of $\varphi^{\prime}{ }_{s}$. The norm of violations of conditions (21), (23) was taken as error evaluation for the initial problem and its accuracy 0.1 appeared sufficient for computation.

We illustrate the work of the method with two examples. The first model contained five auction markets. Each market involved one buyer. The numbers of sellers were the following: market $1-108$, market $2-63$, market $3-48$, market 4 22, market 5 - 44; see Fig. (1).

In the picture, $\Delta$ denotes the excess supply at the market, $p$ denotes the auction price at the market, these values are given in parentheses. Also, the numbers in brackets denote the upper bounds for direct and reverse (-) flows, one number corresponds to the case of symmetric bounds. The given solution was obtained in 22 proximal iterations.

The second problem corresponded to the network with 19 nodes, containing auctions with 106 sellers and 7 buyers; see Fig. (2). The solution presented in Fig. (3) was obtained in 280 proximal iterations.

In all the cases, the solution time for the processor Intel Pentium $1.73 \mathrm{GHz}$ did not exceed one second. Thus, the computational results of the method appeared satisfactory for application.

\section{Conclusions}

In the paper, we proposed the variational inequality approach for modeling both separate and spatial auction markets where the behavior of participants is very complex and mutually dependent. As a result, we can apply the well developed techniques for investigation and solution of these auction market problems. In such a way, we can find both the bid and offer volumes and auction clearing prices for each separate market. Moreover, this approach allows us to easily obtain existence and uniqueness results and develop various iterative solution methods. Taking into account peculiarities of the models, in particular, the simple structure of constraints, makes these methods rather efficient. Preliminary results of computations showed rather fast convergence to a solution together with very simple implementation. 


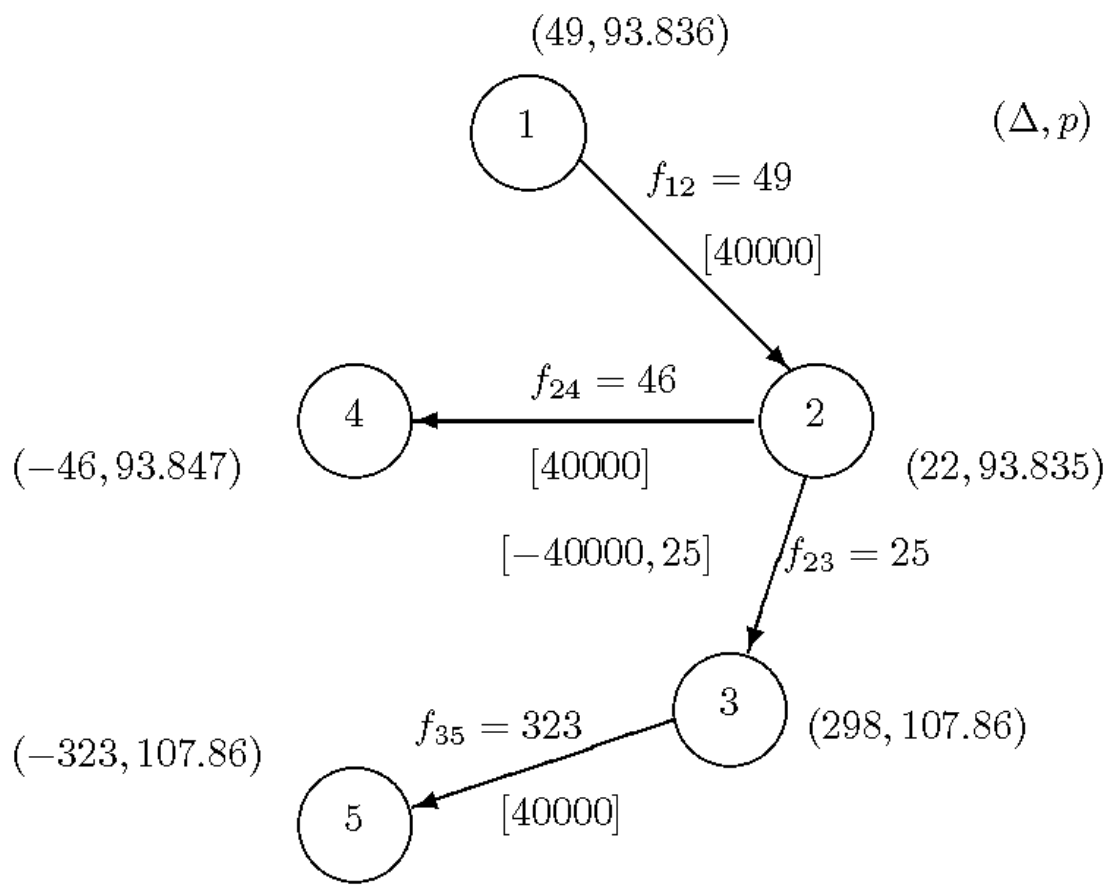

Fig. (1). Spatial market 1: Data and solution.

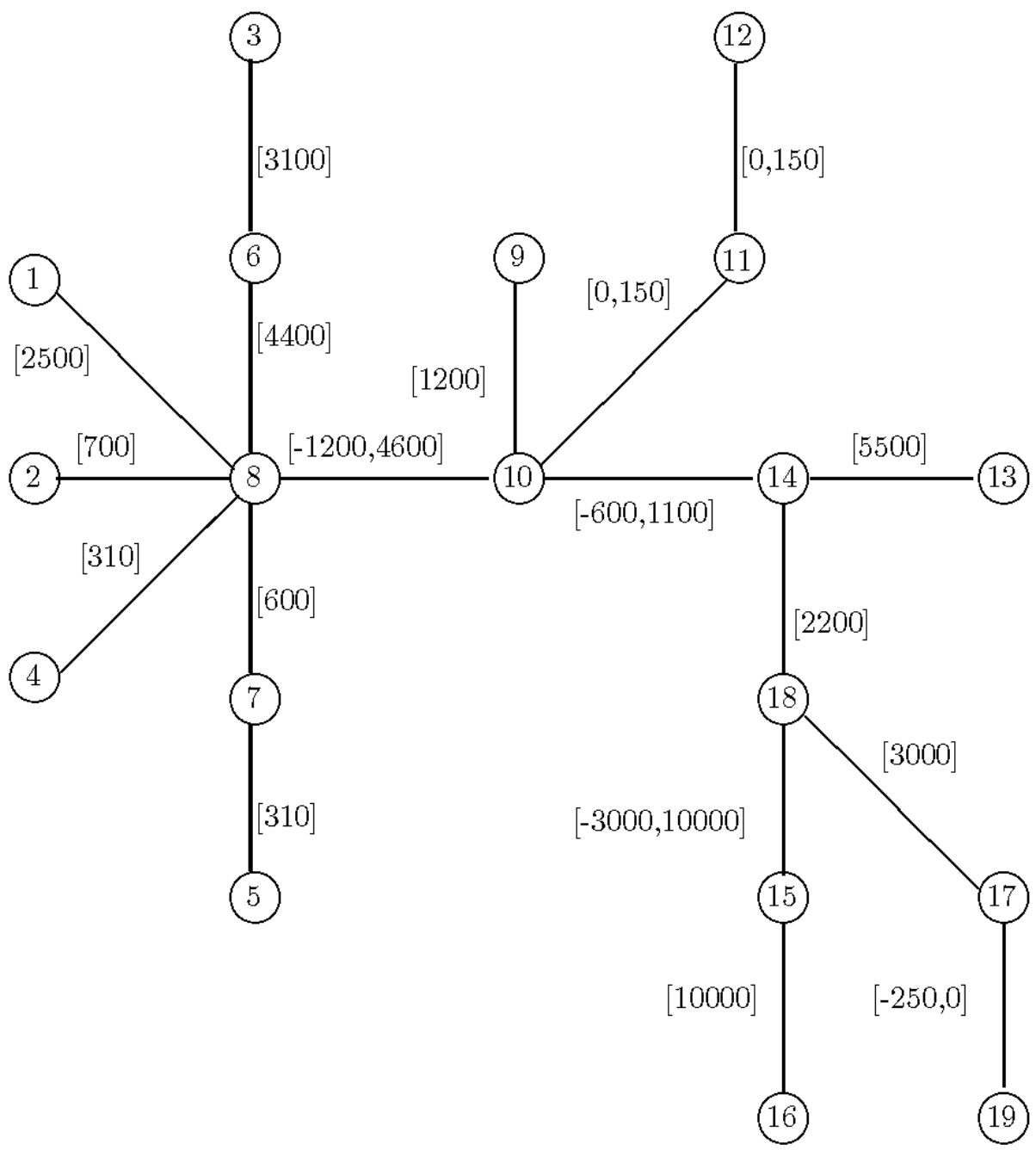

Fig. (2). Spatial market 2: Data. 


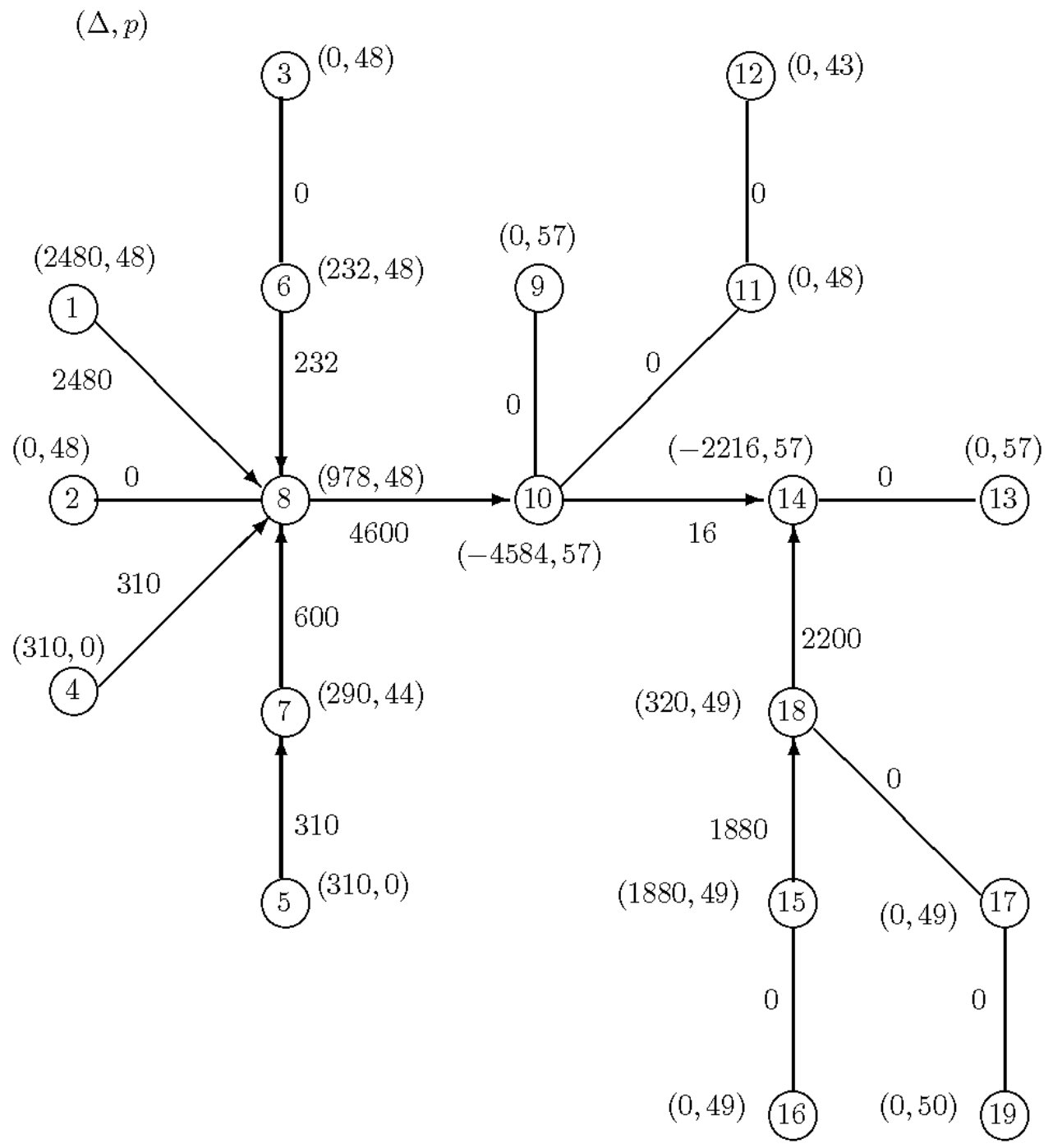

Fig. (3). Spatial market 2: Solution.

\section{ACKNOWLEDGEMENT}

In this work, the author was supported by RFBR Grant No. 07-01-00499.

\section{REFERENCES}

[1] Moulin H. Théorie des jeux pour l'économie et la politique. Paris: Hermann 1981.

[2] Weber RJ. Auctions and competitive bidding. In: Young HP, Ed. Fair allocation (Proceedings of Symposia in Applied Mathematics, Vol.33). Providence: American Mathematical Society, 1985; 14370.

[3] Konnov IV. On modeling of auction type markets. Issled Inform 2006; 10: 73-6 (in Russian)

[4] Konnov IV. Equilibrium models and variational inequalities (Mathematics in Science and Engineering), Amsterdam: Elsevier, 2007; Vol. 210.

[5] Konnov IV. On variational inequalities for auction market problems. Optimiz Lett 2007; 1: 155-62.

[6] Metzler C, Hobbs BF, Pang J-S. Nash-Cournot equilibria in power markets on a linearized DC network with arbitrage: formulations and properties. Netw Spat Econ 2003; 3: 123-50.
[7] Beraldi P, Conforti D, Triki C, Violi A. Constrained auction clearing in the Italian electricity market. 2004; 4 OR 2(1); 35-51.

[8] Sukharev AG, Timokhov AV, Fedorov VV. A course in optimization methods. Moscow: Nauka, 1986 (in Russian).

[9] Facchinei F, Pang J-S. Finite-dimensional variational inequalities and complementarity problems. Berlin: Springer-Verlag, 2003.

[10] Fang SC, Petersen EL. Generalized variational inequalities. J Optimiz Theory Appl 1982; 38: 363-83.

[11] Isac G. Complementarity problems. Berlin: Springer-Verlag, 1992.

[12] Konnov IV. Combined relaxation methods for variational inequalities (Lecture Notes in Economics and Mathematical Systems), Berlin: Springer-Verlag 2001; Vol. 495.

[13] Patriksson M. Nonlinear programming and variational inequality problems: A unified approach. Dordrecht: Kluwer Academic Publishers, 1999.

[14] Rockafellar RT. Monotone operators and the proximal point algorithm. SIAM J Control Optim 1976; 14: 877-98.

[15] Ibaraki S, Fukushima M, Ibaraki T. Primal-dual proximal point algorithm for linearly constrained convex programming problems. Comput Optim Appl 1992; 1: 207-26.

[16] Rockafellar RT. Network flows and monotropic programming. New York: John Wiley and Sons, 1983. 
[17] Tseng P, Bertsekas DP. Relaxation methods for monotropic programs. Math Progr 1990; 46: 127-51.

[18] Gol'shtein EG, Tret'yakov NV. Modified Lagrange functions. Moscow: Nauka, 1989 (in Russian; Engl. transl.: Modified Lagrangians and monotone maps in optimization. New York: John Wiley and Sons, 1996).
[19] Kaplan A, Tichatschke R. Auxiliary problem principle and proximal point methods. J Global Optimiz 2000; 17: 201-24.

[20] Azhmyakov V, Schmidt WH. Strong convergence of the proximalbased method for convex optimization. Math Method Oper Res 2003; 57: 393-407.

(C) I.V. Konnov; Licensee Bentham Open.

This is an open access article distributed under the terms of the Creative Commons Attribution License (http://creativecommons.org/licenses/by/2.5/), which permits unrestrictive use, distribution, and reproduction in any medium, provided the original work is properly cited. 